\title{
BRScS Approach for Resolving Heterogeneity of Data from Multiple Resources at Semantic Level
}

\author{
Muhammad Farhan Ramzan $\left(\mathbb{D},{ }^{1}\right.$ Zaigham Mushtaq ${ }^{(D},{ }^{1}$ Sikandar Ali $\left(\mathbb{D},{ }^{2}\right.$ Ali Samad, \\ Mujtaba Husnain $\mathbb{D}^{1},{ }^{1}$ and Mukhtaj Khan $\mathbb{D}^{2}$ \\ ${ }^{1}$ Faculty of Computing, The Islamia University of Bahawalpur, Bahawalpur 63100, Pakistan \\ ${ }^{2}$ Department of Information Technology, The University of Haripur, Haripur 22620, Khyber Pakhtunkhwa, Pakistan \\ Correspondence should be addressed to Sikandar Ali; sikandar@cup.edu.cn and Mukhtaj Khan; mukhtaj.khan@awkum.edu.pk
}

Received 31 December 2021; Accepted 27 January 2022; Published 3 March 2022

Academic Editor: Ewa Rak

Copyright (c) 2022 Muhammad Farhan Ramzan et al. This is an open access article distributed under the Creative Commons Attribution License, which permits unrestricted use, distribution, and reproduction in any medium, provided the original work is properly cited.

\begin{abstract}
Data have multiplied at an exponential rate in the age of the Internet. Large amounts of data can be combined at this science hotspot. Making sense of big data has become increasingly difficult due to its volume, velocity, precision, and variety (sometimes referred to as heterogeneity). Many data sources are employed to create data heterogeneity. Big data fusion has both advantages and disadvantages when it comes to integrating data from a variety of sources. The focus of this work is on large data fusion using deep learning approaches to combine datasets from a variety of different sources. It is also possible to combine data from many sources. People are increasingly turning to the Internet and web-based services to meet their daily demands. Storage media can hold data in a variety of formats. Managing the vast volume of data is quite tough for an organization (referred to as "big data"). These data are rationally combined and incorporated into the system. Data fusion will be the subject of this paper. The process of collecting data and making judgments based on that data has become much more challenging as a result of technological advancements. The heterogeneity of data is made possible by the great volume, precision, and, most critically, variety of big data. A wide range of data sources can both help and hinder big-data converging. This study was created to introduce several methods and techniques for semantically merging huge datasets.
\end{abstract}

\section{Introduction}

Intelligence is critical in intelligent warfare. As many devices improve, especially the performance of the multisource nonuniform sensor, more multisource nonuniform information data are generated [1]. Based on the battlefield and the target's qualities, these data can be used to define the target's activity rules. I can learn how to combine battlefield data using the tools I already have. The military situation is predicted as shown in Figure 1, as well as its trend and aim. Figure 1 shows proper military condition. Because of the high-level characteristics of battle environmental objectives, the mining sector can more correctly estimate the target method. It is possible to validate, match, and explain this information so that reliable predictions and choices may be made about the activities of battlefield targets. Global competition to develop new military technologies is at an alltime high. Deep abstract features can be used to describe the target's properties, for example, by the target's high-level function. As a starting point, these characteristics might be utilized to characterize the goal. When employing the target function's technique [2], the selection of a function manual is simple. However, in order to be more universally applicable, the selection object functions in the manual tend to be shallow, with minimum feature abstraction and constrained semantic expression. Deep learning, a newly developed technique for extracting object depth features, performs remarkably well. To put it another way, deep learning has had a dramatic and considerable impact on the target height function. It is critical to know how these variables interact, both as a direct threat on the battlefield and as a complex, abstract data source. Target extraction and selection for 


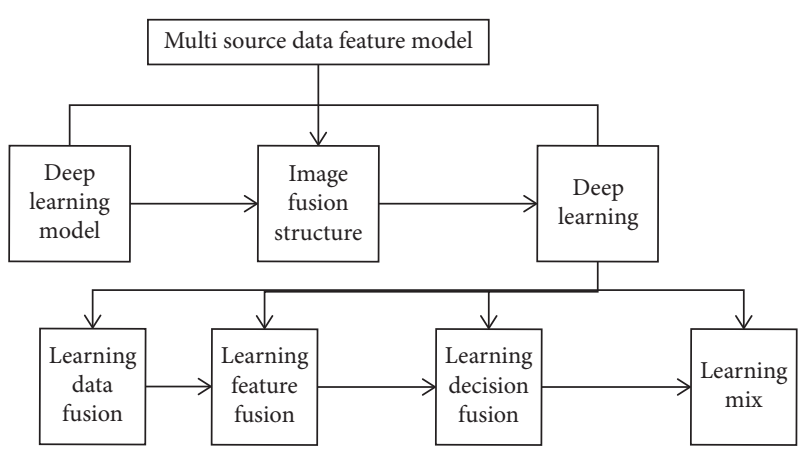

Figure 1: Model building.

high-level targets are done using in-depth learning based on target extraction and selection. The ultimate goal of technical research is to create activity rules. The activity trend forecast they mine is the most basic technology for real-time and accurate updates. The realistic fighting scene may help in finding rather new information.

Data sources and data types are evolving at a rapid rate as a result of technological advancements. Various technologies, such as complex data structures and highly multidimensional data, have resulted in numerous types of big data being generated today. The rapid speed of change makes traditional data storage and extraction increasingly challenging. Data structure heterogeneity can be found in structured, semistructured, and unstructured datasets. A wide range of data is included, such as facts about people, places, things, and the universe itself. Sensor data, for example, are the major source of information that needs to be combined.

The heterogeneity of the data makes collaboration and crosspollination, and the integration of heterogeneous data from multiple sources is difficult. There is a new activity in the great data age where the new characteristics of the great data age meet heterogeneous data fusion from different sources. To get the best results, computer data from a variety of sources must be analyzed.

The integration and fusion of data from various sources are a key technology supporting the genome materials initiative (Figure 2). New information about materials with unique properties is discovered by combining data from experiments and industry. This initiative's overall goal is to double global and domestic production and use rates of cutting-edge material technologies. Former President Obama stated this in his inaugural address. In order to discover new materials and improve the production of existing ones, data from various sources will be combined. The material data's multisource property makes use of information gleaned from numerous sources. There is always a wide range of data formats, definitions, quality levels, and processing methods in multisource material data. A material is a substance that can be used to create useful objects, such as tools and other equipment. Making things is critical to the advancement of human society as a whole. The study of materials has ramifications in virtually every branch of science and engineering, including biology, chemistry, physics, and engineering. Microstructure and

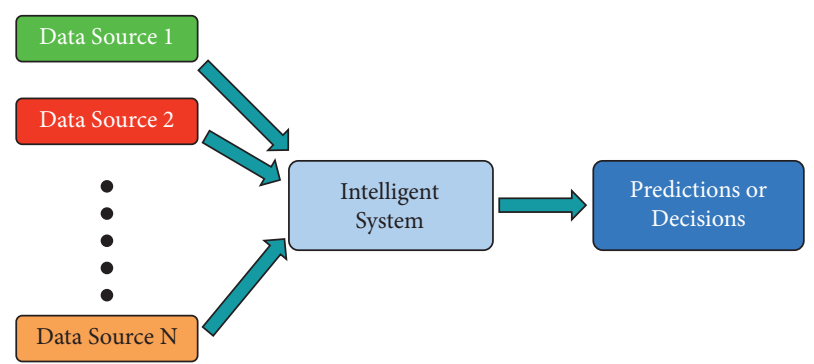

FIgURe 2: Prediction based on Integration \& data fusion.

macroperformance of materials, as well as science and technology, are all studied in materials science.

Theories in the fields of materials science include those from many other fields as diverse as chemistry, biology, electrical engineering, and informatics as well as solid-state physics. Integrating materials science into other fields will almost always lead to new discoveries and new materials, as is generally agreed. Electronic materials can benefit from a combination of material science and electronic engineering, whereas structural materials benefit from a combination of material science and mechanics. New rules based on heterogeneous multisource materials have been discovered because information about material structure, molecular matter formulas, and chemical reactions are all included in multisource material information.

A test mission, on the other hand, does not involve the combination of data from various sources. Heterogeneous data from various sources, such as underlying data, semiprimary data, and unstructured data, can be organized in a variety of ways, generally speaking. Prior to merging heterogeneous data from multiple sources, it is necessary to design a structure that depicts the data as a whole. Material data are distinct from other types of data in a number of ways. There is a lot of inconsistency in the data. Material data sources are also a risky proposition when it comes to working semantically. Examples of high-dimensional, inconsistent material data include those in three dimensions, two dimensions, and even higher dimensions. Many sources of data must be brought together into a single system in order to perform data fusion. The accuracy of the content ontology mapping is another issue. For a single chemical, the scientific name may change over time and across different languages. Chemical linguists use a lot of abbreviations and shorthand. Consider how difficult it is to deal with data that is encoded and formatted in a variety of ways. Conflicts over multiple sources of content data must be resolved quickly if they are to be avoided.

Current research is focused on fusion learning and multisource, heterogeneous data feature construction [3]. This paper proposes an effective and reasonable feature fusion strategy in order to extract complementary information from different feature layers in order to increase the model's flexibility and scalability. The combination of a deep information fusion model and a neural network for inference, deep learning, training, and learning is based on multisource fusion mode for heterogeneous data input, fusion of multisource sharing function for feature extraction 
of high-level target features. A simple and generalized deep learning framework is constructed by first extracting the target's multivalued features from the data source. We investigate the framework's feature transformation, selection, and classification methods in depth in order to build a powerful deep feature model. Research into the model's feature transformation and classifier methods, along with its core algorithm, is being carried out in order to better utilize its model-learned characteristics and those of each layer. To extract the advanced function of a selected object, the deep learning model is used in conjunction with a feature transformation algorithm and a feature selection method. Deep feature learning mode, deep learning construction mode of multisource data intellectuality, and the general feature vector can all be used together as a third method. It is possible to apply the hybrid heterogeneous data fusion method to virtually any single model [4] by using an allencompassing deep learning mode and a unified learning model. Learning the fundamental components of a global deep learning model such as a composite model of input data and simultaneous training combination is essential. Data fusion models that use multiple sources of data can greatly benefit from this approach. The multisource fusion deepseated model can be implemented using feature learning and composite feature extraction methods from a deep-seated model. Research begins with the development of a hierarchical structure, which should be built using a deep learning model. To put it all together, the framework is made up of feature learning modules with multiple levels and classification modules at the end. A simple deep learning approach used for feature extraction, feature selection, and feature classification must be able to generalize well enough to begin building a model [1-6].

\section{Characteristics of Big Data}

2.1. V's of Big Data. The rapid expansion and innovation of the Internet have resulted in a new era of massive information on the Internet. Massive amounts of data have the following four characteristics. There is a lot of information, and it is presented in a variety of ways. It is the sheer volume of large data that is mind-boggling, as it indicates an unsteady collection of information. Although Flicker produces just over 3 trillion bytes of data, Google produces over 20 trillion bytes of data. Massive amounts of data are being generated at an alarming rate, necessitating ongoing planning, according to the statement that the pace is enormous. Online help services require a lot of data, so businesses on the Internet have to keep a lot of information on hand. One of the main characteristics of massive amounts of data is the presence of a large number of erroneous or redundant data elements, such as noise, errors, and incomplete objects. The diversity, or variability, of big data is unquestionably its most distinguishing feature. The database contains 75 percent structured data and 25 percent unstructured data.

As a result of the wide range of data collection equipment, the data collected is frequently of various kinds. Big data have a significant impact on data integration and analytics.
2.2. Multisource Heterogeneous Data. A wide variety of data types are protected in the same way that multimodal data are. As a model, the existence of different data types (text, image, and video) can be interpreted. On websites like Facebook and other social media, images are frequently accompanied by text. As a multimodal and heterogeneous dataset, it is important to note. Structured, semistructured, and unstructured data all fall under the umbrella of multimodal information. It is critical to have a data fusion strategy that takes into account a variety of different types of data. Heterogeneous data derived from multiple sources can help fill in the gaps when a single data source is unable to provide complete information on the target item. Once the ambiguity of heterogeneous data is removed and separate data sources are combined, it becomes more valuable [7-16].

\section{Big Data Fusion}

The fusion of data is a necessity for the assurance of consistency and analysis of integrated data. Data fusion as a whole however is a black-box operation for the consumer that lacks interpretability and debugs capabilities for the data fusion process [17-19].

There are three types of data fusion: fusion at the data layer, fusion at the capacity layer, and fusion at the choice layer. It is possible to further break down each of these groups into even more specific ones. Data layer fusion refers to the low-level reconciliation of the first-level data. When two or more elements are fused at the atomic level, this is referred to as a "centre level fusion." Once the initial data have been separated, it is artificially reviewed and processed. Constant research is made easier with this system because it keeps the informational pressure high. Each step in the decision-making process uses data from multiple sources, including the pretreatment, feature extraction, identification and discrimination, and preliminary findings. Once the correlation has been processed, the decision-making fusion takes place and the expected outcomes are obtained. Image data, audio data, and Chinese text all need to be combined into a single piece so that the multisource information's heterogeneity can be comprehended. The data layer integrations require homogeneous and isomorphic data [20]. It is also through heterogeny that heterogeneity is developed, and vice versa. For multisource, heterogeneous data fusion, the most important difficulty is determining how various data will work together to accomplish a goal, such as creating or locating task definitions or locations [21-25].

\section{Multisource Heterogeneous Data Fusion}

System of sensors or multiple sources for the fusion of information from multiple sources is a common practice in information processing. There are a number of ways in which they can improve their ability to make accurate predictions, including the use of techniques like data aggregation, data integration, and data filtering. During World War II, a multisource synthesis was developed for military purposes. At 
first, it was primarily used by the military, but it has since been adopted by a wide range of other sectors. The processing object is derived from a variety of sources, including sensor data. A key component is a scalar information (also known as information fusion multisensor technology).

One of the most important aspects of big data is its heterogeneity. Because of the variety of formats and modes in which they are represented, data from numerous sources may be linked, intertwined, and inconsistently interpreted. When dealing with large amounts of big data, structured, semistructured, and even completely unstructured data must all be collected and analyzed at the same time. Using the term "big data" refers to a set of discrete datasets that contain a large amount of data and dynamic information. Because it often necessitates the collection and storage of data that is based on numerous criteria or patterns, big data analysis can be difficult.

There is a need for a greater understanding of the context [18]. For example, existing manufacturing data does not correspond to the user's history, timing, behaviors, tasks, and location. With enormous datasets, contextualization can be an attractive paradigm to mix disparate data flux to increase the effectiveness of a mining process or classification. Big data operations (for example, cyber-physical monitoring for real-world circumstances) can be reduced in resource consumption if they are concentrated on a single source, as has been proved to work well.

\section{Literature Review}

Zhou et al.'s study [17] contains a substantial amount of material data, mainly from multiple sources, with varying data formats and semantic links. The inclusion and convergence of material information can help with material disambiguation, material attribute extraction, standardization of material occurrences, and material security. Nevertheless, new opportunities for material information gathering are presented by rapid data innovation, such as artificial reasoning and massive data. No comprehensive study of modern material information combination processes has been done in our area. First, this paper examines the nature of material data and the reasons for combining data from multiple sources. After that, we will look at the most recent developments in multisource data fusion. The findings of this study are quite different from those of previous research in a number of ways. The manufacturing flow of material data is a formal classification and comparison method for material data fusion. Artificial intelligence algorithms and big data techniques for material fusion will also be discussed. This section concludes with a list of open questions and possible research avenues.

According to Zhou et al. [19], a new era of big data is upon us, as the volume of data generated by the Internet continues to soar. Data fusion generates enormous value and is a research area. In the age of big data, information has increased in volume, speed, accuracy, as well as heterogeneity. Data heterogeneity is caused by a wide range of data sources. Heterogeneous data from various sources complicate the fusion of large amounts of big data. This study examines big data fusion and heterogeneous data fusion from a multisource perspective. In addition, there is a discussion about how to deal with heterogeneous data.

In the words of Zhang et al., remote sensing technology is constantly evolving, and new methods and algorithms are needed to keep up. Fusion technologies have been developed in a wide range of fields, including satellite Earth observation, computer vision, medical image processing, and defense protection, among others. It is difficult to combine data from multiple sources because of the wide range of geographic and temporal resolutions available. This article examines current strategies for distant data fusion from multisource sensing based on the hierarchy concept and looks ahead to potential developments and issues. Panchromatic and multispectral data fusion is discussed in this article. In high-level pixel fusion techniques, multisource data at the feature level, including synthetic aperture radar, LiDAR, and other data formats, are used. A few developments in multisource data fusion are summarized in this article.

This article [27] is a good place to start. For the past two decades, doctors and health-care providers have relied on images to describe the human body's normal and abnormal structures. A single imaging mode is not enough for medical experts to study and examine knowledge effectively, however. With this approach, clinicians can identify and treat medical issues at an early stage, increasing their understanding of both qualitative and quantitative aspects of health care. For multiresolution image registration, MRR uses DWT for picture fusion and PCA for principal component averaging. Additionally, the proposed DWT-PCAv combination method contributes more useful information with less computation time than the proposed MRR strategy alone, which is more reliable. Brain CT and MRI scans from the HARVARD dataset are used to test the new method proposed. There are fusion effects present in the proposed method when compared to current fusion approaches. For statistical comparison of quality ratings, the mutual information (MI), crossnormalization correlation (NCC), and mutual information feature (MIF) are all computed (FMI). Using this method, we can get better results, better images, and more accurate medical diagnoses.

This is an in-depth look at the topic. [28] Traffic monitoring is made easier by the availability of multiple freeway data sources, but problems can also arise. Researchers in this study combined the use of microwave sensors with that of current cellular handoff sensors. Fusion of neural networks was also introduced. Fusion model inputs were tweaked to see how these factors affected the model's ability to correctly predict outcomes. Handoff length and sample size were found to be the most important factors in fusion accuracy, as demonstrated by the study results. The proposed fusion system was then tested in a variety of traffic scenarios to determine its effectiveness and capability. Other approaches have been contrasted with this one. It has been determined that fusion methods will improve the overall estimation accuracy, adjust data to changing traffic conditions (free flow or incident state), and adapt to other fusion methods of cell phone and fixed sensor data, as well as execute other fusion methods. 
We came up with a model for the fusion of heterogeneous data from multiple sources. In order to give an express multidata definition, we used three-way fussy numbers and the ordered weight average (OWA) for preference determination and algorithm design. An actual-world example, at long last, proved its value. [29].

Ariz-I and colleagues' recommendation systems can also help alleviate the problem of Internet overload. Combining data from multiple sources allows for more accurate recommendations. Many people believe that modern fusion recommendation models combine the conclusions of multiple sources of data. Their immediate and indirect friends tend to have an impact on what users like. The BRScS hybrid recommendation model is proposed in this study (an acronym for BPR-Review-Score-Social). A normal depiction learning framework uses societal data, scores, and analysis to compile the top $\mathrm{N}$ proposals. Using a PV-DBOW model to process the survey data, and a fully connected neural network to process the rate data, the PV-DBOW model can be used to combine and decompose social relationships. On the Howl public dataset, the BRScS proposed calculation outperforms BRSc, UserCF, and HRSc. New data structures can be easily incorporated into the BRScS paradigm.

(Text at 30) In the field of space information technology, multisource and heterogeneous data integration was a problem. This study examined the advantages and practicality of using ontologies to evaluate all related integrating procedures in order to overcome the semantic challenge. This study developed a platform for ontology data integration by first building a network and then using that platform to integrate data. Every facet of the investigation was thoroughly examined.

Because of opacity, light fluctuations, and dynamic background, traditional optical pedestrian surveillance systems [20] fail in this study. These problems can be solved by obtaining more sensitive information. Multiple sensor data streams must be combined for accurate tracking. Film, RGB-D, and inertial sensors are being used to construct a foot tracking system. By merging texture and depth features in an RGB-D sequence, we are able to locate the desired object. The inertial sensor positioning error is eliminated by using the local location. For accurate tracking, a camera calibration method uses a video picture plane map to map the inertial sensor's location, which is then combined with the visual monitor's position. Real-world studies have shown that the built-in tracking approach beats the current singlesensor tracking method and is immune to occlusion, illumination shifts, and interference from similar textures or circumstances.

Narrow-band Internet of Things (IoT) has emerged as the de facto standard for IoT due to its extensive reach, low power consumption, low cost, and low latency [18]. Nevertheless, with so many terminals, the allocation of resources and heterogeneous data fusion within the scheme becomes an important issue in Internet of Things research. So, this study proposes a semantic data fusion for the NB-IoT. To begin, let's take a look at the advantages and key features of NB-IoT, including the physical and media access control layer techniques that are most important. In order to produce a single file format, we use semantic senses to combine data from multiple sources and heterogeneous sources. We use an improved D-S theory for the ultimate fusion effects. It appears that our approach has a faster convergence rate, better stability, and a higher degree of accuracy in the evaluation of fusion results.

\section{Deep Learning Model}

"Feature transformation nonlinear operation feature selection (subtraction)" [5] is a common deep learning model. In this stage, you can design the feature transformation of the filter and other feature extraction methods. The nonlinear operation can also use the logistic regression function to double the transformation's properties. Both active and forbidden states for classification and other machinery are used. The learning topics' qualities are also selected. The size is also smaller. As a result, the deep network model's scalability is limited. The distributed feature representation of data is discovered by mixing the low-level deep learning characteristics explored by artificial neural networks. Deep learning is stacked like neural networks. The system has three layers: input, concealed (multilayer), and output. Only nearby layer nodes are linked. It matters not.

There are different techniques to extract data sources and advanced object features. The problem of feature extraction for various data sources emerges under the unified framework of information fusion technology. The data fusion model is based on the level of data abstraction in common data, and the data extraction level is based on the feature layer fusion. We propose a hybrid fusion of machine learning flexibility and heterogeneous data fusion complexity. An investigation of information fusion architecture for light, SAR, and infrared pictures is offered. Pixel fusion can be done without modifying data [8]. In a multisource heterogeneous image, the heterogeneous camera mechanism's differential image data are changed, making pixel level (horizontal) fusion hard. Three-pixel fusion models are explained: decision fusion, feature fusion, and hybrid fusion. Target state and target feature information fusion are two types of feature fusion. Target state fusion is used for tracking. Parameter correlation and state vector estimation are applied in fusion. Multisource data include intelligent radar, tracking infrared, and other nonimage data. The fusion of target features is widely understood. The fusion approach is closely similar to pattern recognition technology, although it is a merged characteristic. In addition to intelligent radars, various nonimaging data sources are involved. The image sources for SAR, infrared, and visible light are all described. Infrared and SAR images are combined with information about the target's optical qualities and infrared measurements. A fusion structure is shown in Figure 3. Combining many sensor images into a single feature extraction process might increase the likelihood of obtaining high-quality composite features.

A neural network model is created after developing a training plan. Basic neurons make up the neural network, and each one has an output. Figure 1 depicts the net in the form of a circle. A " +1 " circle denotes the intercept period's 


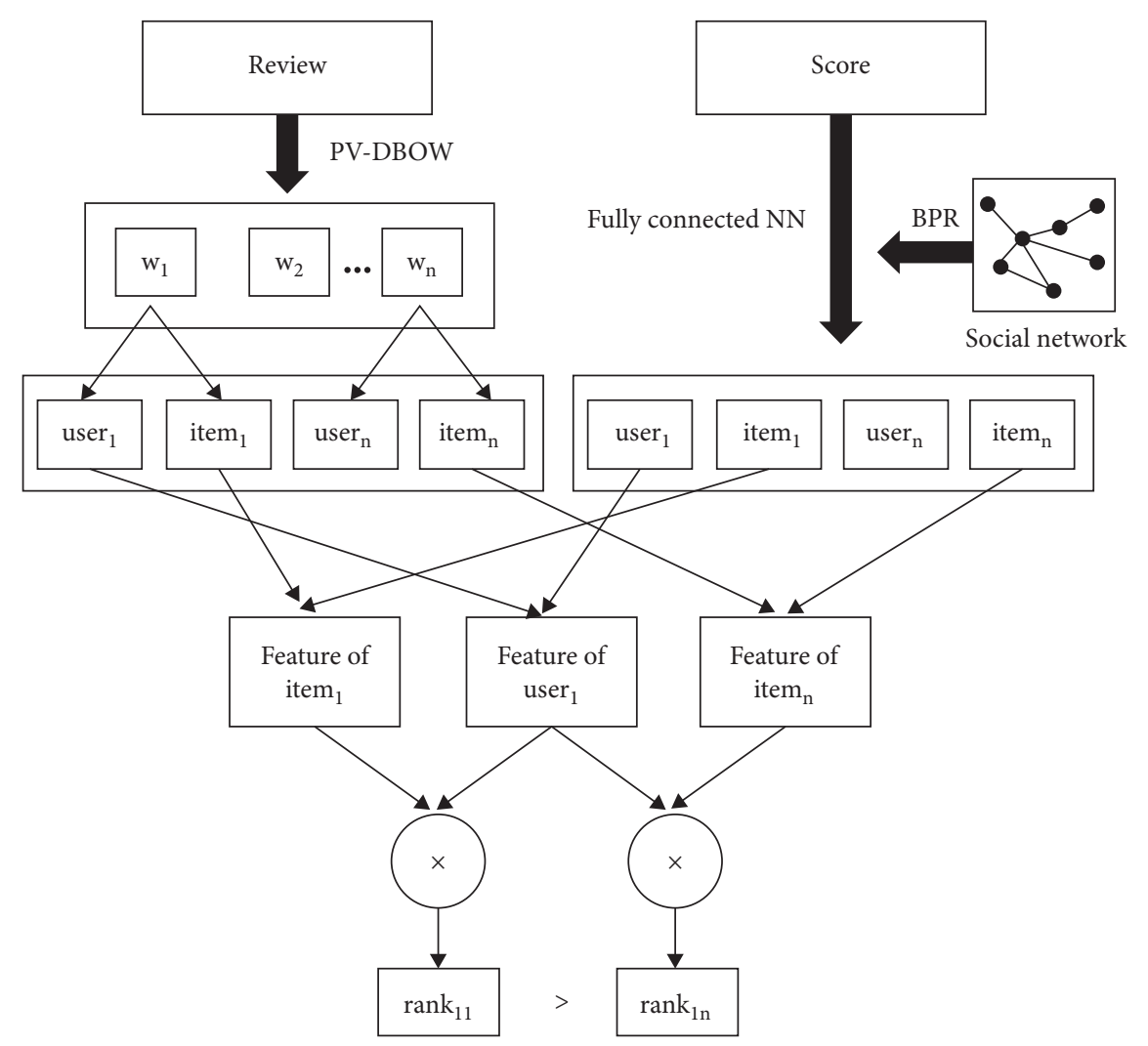

FIgURE 3: Recommendation process.

offset unit. The top layer of the network is the input, while the bottom layer is the output. Most people do not know anything about it. It is not possible to see the value of the node in the training data [6]. There are three units (without the bias device) in the figure's artificial neural network's input layer, each with two hidden layers. The three hidden components of the first layer and the two hidden parts of the second hidden layer can be used to define complex nonlinear hypotheses. Necessary alterations are made to $\mathrm{W}$ and $\mathrm{B}$ in order to achieve your objectives. This is an improvement to the training procedure. For the network to be properly trained, we need examples to draw from the study of et al [7]. However, the real-world network model is a lot bigger than this. The proposed neural network model has 16 layers as a result of convolution, convergence, and complete connectivity. Linear functions are used by neurons. Slow ball is finalized. Convolution and convergence are essential to deep neural networks. An analytical concept is based on the human visual receiving field, with the emphasis in the middle. The nature of an overlay changes as the image changes its two-dimensional space. You can acquire new skills that will be beneficial in the future. It is possible to reduce the number of parameters in a convolution kernel by sharing it. Convergence is used to downsample images and to learn spatial properties. It is possible to change these functions and rotate them. A cost function $J(\mathrm{~W},(\mathrm{~b})$ is defined as the average square differences in cost. The weight of your adjustment items is minimized to avoid overlapping them.
All nodes in the network must be able to use the network model. Training samples are required for this. Iteratively correcting network parameters is the goal of each training session. For as long as training samples are diverse, the network can recognize and learn new items. Object representations are formed as a result of cognitive learning. Detection accuracy has increased as well as target learning has become more thorough and characteristics more distinct. As a result, optimizing the model parameters is crucial.

There are a variety of methods for obtaining data sources and enhancing object features. Because of the unified structure of information fusion technology, it is difficult to extract features from different data sources. Because the amount of data abstraction in common data is high, the data fusion model relies on the feature layer fusion to extract useful information. Machine learning adaptability and heterogeneous data fusion complexity are combined in a hybrid approach that we present here. For light, SAR, and infrared pictures, an information fusion architecture is described. It is possible to perform pixel fusion without modifying data [8]. Because of the transformation of heterogeneous camera mechanisms' differential images data, horizontal fusion is difficult in multisource heterogeneous images. Models that combine different types of pixels into one image are discussed: decision, feature, and hybrid. Fusion of target state and feature information is one type of feature fusion, whereas fusion of target features is another. Tracking is done using target state fusion. Fusion makes use 
of parameter correlation and state vector estimation. Infrared and other nonimage elements are also included in multisource data. Target qualities are well understood. Although it has several characteristics with pattern recognition, the fusion approach incorporates both. Other nonimaging data sources are included in addition to intelligent radars. The image sources for SAR, infrared, and visible light are all described. Infrared and SAR images are combined with information about the target's optical qualities and infrared measurements. A fusion structure is shown in Figure 3. The probability and accuracy of feature extraction can be improved by combining multiple sensor images.

\section{Data Level Fusion Based on Deep Learning}

Data level fusion is a term used to describe the merging of pixels in an image. There are numerous approaches to merge images from multiple sensors in the same space-time context, such as employing pixel fusion. It has the capacity to incorporate a wide range of images. Deep learning data fusion uses these images of unequal fusion as training samples. The final classification and identification results can be generated using a neural network model that can recognize a target in a picture with uneven fusion. 6. End of an era deep learning has had a profound impact on the field of image and sound research. Rather than traditional vector features, the most advanced model of deep learning makes use of two-dimensional spatial meaning information instead. A multisource, heterogeneous data fusion paradigm is also missing. A multisource deep learning system is employed in this article.

In this study, a deep learning-based recommendation engine that can evaluate score, review, and social data is offered.

Lack of data and inadequate precision in the score are the main limitations of classical matrix decomposition. An artificial neural network is used to turn scores into representations of the user and the item. The usual topic model does not effectively convey the spirit of the reviews. In order to learn feature representations of reviews, the PV-DBOW approach is applied. PV-DBOW uses a document vector model to improve word prediction. Increased accuracy in suggestions can be accomplished by the use of social network data in conjunction with the paired learning approach.

7.1. Making a Recommendation. Due to the multiplicity of inputs, the final suggestion is traditionally formed by combining the findings of various algorithms [30]. Deep learning can accurately represent multisource heterogeneous data such as scores and reviews, allowing for the incorporation of all data sources [30]. We propose a recommendation system based on multisource heterogeneous data that incorporates ratings, reviews, and social network information. It is extremely precise and scalable.

As represented in Figure 2, the proposed procedure is illustrated. A user's entire happiness with a product is expressed by the score. Multilayer neural networks directly learn the feature vector representations of both users and items. A review is a person's appraisal of a product or service, and it may be a significant source of information for both the consumer and the product. Using the PV-DBOW approach, the user and item feature vector representations may be learned. User friendships are reflected in the social network. Users' selections will be influenced by their friends' preferences. Predicting future purchases with the usage of social media is conceivable. Improved recommendation accuracy can be achieved with the use of the Bayesian personalized ranking $(\mathrm{BPR})$ model.

7.2. Example of Sound Guidance. Using heterogeneous data from multiple sources, a recommendation model can be built. The user and item triplet optimization models should be created. The social connections from the web are taken and mix them with ratings. Finally, deep learning is used to extract user and item features. In the end, feature representations of users and things provide a top-N list of suggestions. Below is a breakdown of the model.

7.3. User-to-Machine. Friendships can be expressed through social media. People are more inclined to purchase or like things that their friends have purchased for them. As a result, both direct and indirect buddy relationships can be used to predict a user's behavior and preferences in the future.

User preferences are assumed to be shared when using the trust-based method. There is less of an impact on a user's decisions from indirect friends than direct friends. In the 6 degrees of separation concept, user similarity is defined as (1):

$$
\begin{aligned}
\mathrm{s}(\mathrm{a}, \mathrm{b}) & =0.2 \times(6-\mathrm{lab}) \\
\text { In this case, } \mathrm{s}(\mathrm{a}, \mathrm{b}) & =0.2(6 \mathrm{lab}) \text { if lab60.1 is true, else }
\end{aligned}
$$

Any two users can be A and B. When two people are connected by mutual friends, the distance between them is represented as lab, where lab is the distance between their direct and indirect friends. Figure 1 depicts the distance between users.

The distances between users can be used to calculate their similarity. The first-degree friend is the direct friend; the second-degree friend is the indirect friend, and so on. The model is named after an indirect friend with a maximum distance of $6^{\circ}$. Algorithm 1 displays the model's pseudocodes and how to compare users.

Through social networks, it is possible to discover the influence of various acquaintances on a user's decisionmaking.

7.4. Brand-New BPR Design. Reference [28] is an example of BPR. A triplet is created by the user $(u, i, j)$. Triplet: $u$ buys $I$, but $j$ is not purchased. User $u$ likes item I over item $j$, according to this data.

Item $I$ or $j$ is not purchased by user $u$. It implies that the preferences of the user are not known. 
Input: dataset User, social relation data Relation;

Output: similarity Set S;

repeat:

(1): construct a graph by using all users and relations in User and Relation;

(2): calculate lab using igraph;

(3): If $l a b<6$

(4): $s(a, b)=0.2 \times(6-\mathrm{lab})$

(5): else

(6): $s(a, b)=0.1$;

(7): until calculate all users' relations;

(8): return S.

Algorithm 1: 6 Degree model.

User $u$ purchases both $I$ and $j$ from the same vendor. In this case, the user's preferences have been overwritten.

There are two advantages to using the BPR method rather than point-wise learning. To begin, both purchased and unpurchased things are taken into account, with the latter being sorted out last. Another advantage of this model is that it is able to perform well even with limited data.

It is a matrix decomposition ranking technique. In contrast to algorithms such as funkSVD, it optimizes the ranking based on the user's commodity selection. It produces superior results. Figure 3 shows how triplets are made. Plus (+) indicates that $u$ prefers me to $j$. There is a good chance that the user prefers item $J$ over item I.

There is no consideration for social interactions when selecting the BPR triplets [29]. When it comes down to it, people like products that their friends have picked out for them. As a result, BPR model sampling can be based on the similarity between users and their friends. It is possible to boost the precision of suggestion generation by using the triplet with social relation limits that limit the number of people who can be sampled.

User-purchased items (I), non-user-purchased items (J), and user-purchased items (P) are all described as I, J, and P, respectively, and in this example, there are no $D$ items in the system. The user's purchases are referred to as Du. Dp is a list of products that the user's friends have purchased. Du and $\mathrm{DpDu}$ are the user's strong preferences. Since the user is more likely to buy an item DpDu bought by friends than by the user's own preferences, last but not least, $D$ is the least likely product that the user will purchase (DuDp). A training set $T$ can be thought of as a triplet of people and things based on information gleaned from social media networks. Item $j$ is the user's preference over item I in the three-item triplet $(i, j)$. The user or friends of the user purchase merchandise $i$. Item $j$ is an un-purchased item belonging to the user or a friend. Social connections are used to form a user-item triplet.

Prior probabilities must be maximized as part of the Bayesian formula for a suggested list. In (3), the user's preference and the model's parameters are denoted. In (3), the user's preference and the model's parameters are denoted by improving a triplet $(u, i, j$ probability) by changing the model parameters.

Assuming that $(i, j)$ is a pair of independent items, we simplify the expression.
Finally, a model is needed to calculate each item's likelihood of being recommended. Item I is more likely to be purchased than item $j$, according to the sigmoid function used in this model. User-item connections can be modeled using xuij(), which is an arbitrary parametric model. This means that any model that defines the relationship between the user and the item can be used. The new BPR model directly improves the item recommendation ranking. This model is based on PV-DBOW.

The PV-DBOW model learns feature representations of persons and objects based on user and object reviews. If you look at Figure 4, you will see that the model first samples the text and then picks a random word from it. PV-DBOW considers the words in a phrase to be independent of one another and hence requires less storage space for each one of them.

Our model predicts words using paragraph vectors. Following that, each review will be taught to predict words.

7.5. Neural Network with Nodes. The fully connected neural network is utilized to handle the scoring data since it can quickly find optimal solutions [30]. The scoring model's purpose is to minimize the discrepancy between anticipated and actual scores.

7.6. BRScS. We propose the BRScS model for combining multisource heterogeneous data (an acronym for BPR-Review-Score-Social) (see Algorithm 2). It uses an updated BPR model for ranking, a user trust model for social relationships in rating and review data, a PV-DBOW model for review data, and a fully connected neural network for rating data. Finally, an integrated objective function is optimized.

The model optimization objective function is provided as (11) where $u$ is the user's fusion feature representation, and I and $j$ are the item's fusion feature representation. According to the previous definition, the user $u$ prefers item I over item $j$. This paper defines $g()$ as a sigmoid function to calculate the user's various preferences for different things. $\mathrm{g}(u, i, j)=(\mathrm{uTiuTj})$. In the review data, L1 is the objective function, in the scoring data, L2.

$W=W_{1}, W_{2}$ denotes each model's weight parameters. In the review representation learning model, the weight parameter W1 is learned for each user's review. The score 


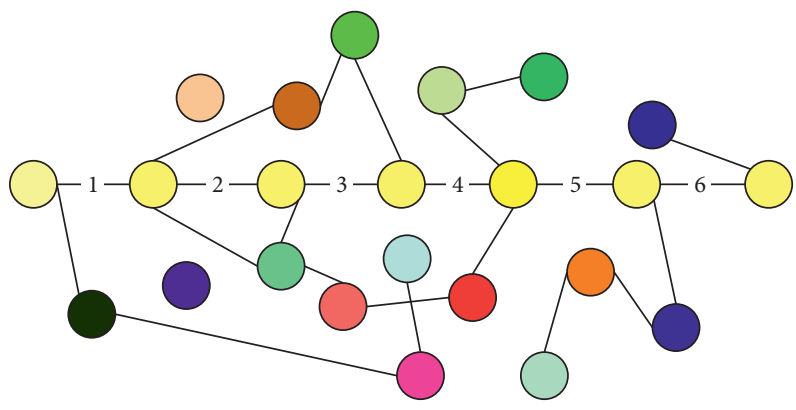

FIGURE 4: Social relations between users.

representation learning model obtains user and item information directly by setting the weight parameter $W_{2}$ to 1 . W2 does not need to be updated by the optimization objective Each model's penalty parameter is in the range $[0,1]$. The score model's objective function $\mathrm{L} 2$ has a negative sign since the score model's objective function should be minimized while the overall model's objective function should be maximized. The stochastic gradient descent (SGD) algorithm is given in the work published in [30] (11).

The higher the $s$, the more likely the user will select the item. A user's top-N recommendation descending from (12).

\section{Results}

A single-core GeForce GTX 1080 Ti running Ubuntu 16.04 is used in two groups of trials. IntelliJ IDEA and Python3.6 are used in the programming environment.

8.1. Dataset. Yelp is an American business directory and review site. It includes restaurants, retail malls, hotels, and tourism. On the Yelp website, users can rate, comment, and share experiences. This study uses the Yelp public dataset from the official Yelp website. The Yelp dataset is JSON and contains user and business details. Data include user and business IDs, user comments and ratings, and user friendship. The user-friend relationship is turned into userfriend pairs. We use user feedback to understand consumer preferences.

To test the suggested model's validity, some users with few comments must be filtered out due to a lack of data. The New-Yelp dataset contains data from the Yelp dataset with more than 20 comments.

8.2. Comparable Test. The experimental results are measured using the following four indicators:

Recall: the proportion of things purchased from the suggestion list to total purchases.

The number of recommended things purchased by users is divided by the total recommended items.

Nominalized discounted cumulative gain determines the rating quality of suggested products.

If the user has purchased the recommended item, it is a hit rate. Buying the recommended item means hits and, otherwise, misses.
Experiment 1. To select a text model and demonstrate the benefits of combining social data with the recommendation, below I compares six models. The most widely utilized text processing models in recommendation systems are HDC and SEL. The HRS model processes review via the HDC, while the SRS model uses the SEL. These models use the BPR framework and PV-DBOW algorithm to process reviews.

The BR (BPR + Review) approach leverages the reviews to provide recommendations.

The BRS (BPR+Review + Social) approach leverages reviews and social network information to make recommendations. The social relation includes both direct and indirect friend relations.

The HRS (HDC+Review + Social) model combines HDC and social network information for the recommendation.

SRS (SEL + Review + Social) model is based on a simplified embedding-based language model. Its recommendations are based on reviews and social media.

BRS $c(B P R+$ Review + Score $)$ model is based on the BPR framework. It recommends based on reviews and scores.

Simplified version of BRS cS $(\mathrm{BPR}+\mathrm{Review}+\mathrm{Score}+$ Social): It combines reviews, scores, and social network information for the recommendation.

The experiment uses 70\% training data and 30\% testing data.

Our hybrid model is BRScS. Figure 5 shows the top- 5 experimental outcomes while Figure 6 shows the top-10 experimental outcomes. The best outcomes are represented in bold script.

Experiment 2. Our model is compared to popular classic recommendation models in Experiment 2.

In order to generate a suggestion, the BRSc model looks at a number of different variables. It is possible to utilize scores as input for UserCF (user-based collaborative filtering algorithm). Reviews and scores are combined in the BRSc model. This is the BRScS model. Experiment findings are shown in Figure 7. Outcomes with the highest likelihood of success are highlighted in bold.

Similarly, Figure 8 represents the results recommended by the top-10 experts of the domain while the performance of the algorithm on different datasets is shown in Figure 9. 
Input: score dataset Score, social relation dataset Relation, review dataset Review, vocabulary V;

Output: user representation $\mathrm{U}$, item representation $\mathrm{M}$, recommendation list $\mathrm{L}$;

(1): Initialize $\theta$, embedding size $=300$, batch size $=64$, negative sample $=5$;

(2): for epoch $=1,2, \ldots, n$ do

(3): split the dataset score, relation, and review into training dataset (70\%) and testing dataset (30\%);

(4): construct positive and negative sample triplets $g(u, i, j)$ based on BPR;

(5): learn the frequency of word-review pair fw, dum and the expected value EWN-PV

(6): get review representation dum;

(7): learn $U_{1}, U_{2}, c_{1}, c_{2}$ from score data;

(8): get score representation $\mathrm{ru}, \mathrm{rm}$;

(9): get distance lab between users;

(10): calculate $\Sigma(\mathrm{u}, \mathrm{i}, \mathrm{j})+\lambda_{1} \mathrm{~L}_{1}-\lambda_{2} \mathrm{~L}_{2}$;

(11): update $\theta=\left\{\theta_{1}, \theta_{2}\right\}$ with back propagation;

(12): get corresponding user and item representations $U, M$

(13): end for

(14): compute s;

(15): return recommendation list $\mathrm{L}$

Algorithm 2: BRS $_{\mathrm{c}} \mathrm{S}$ Model.

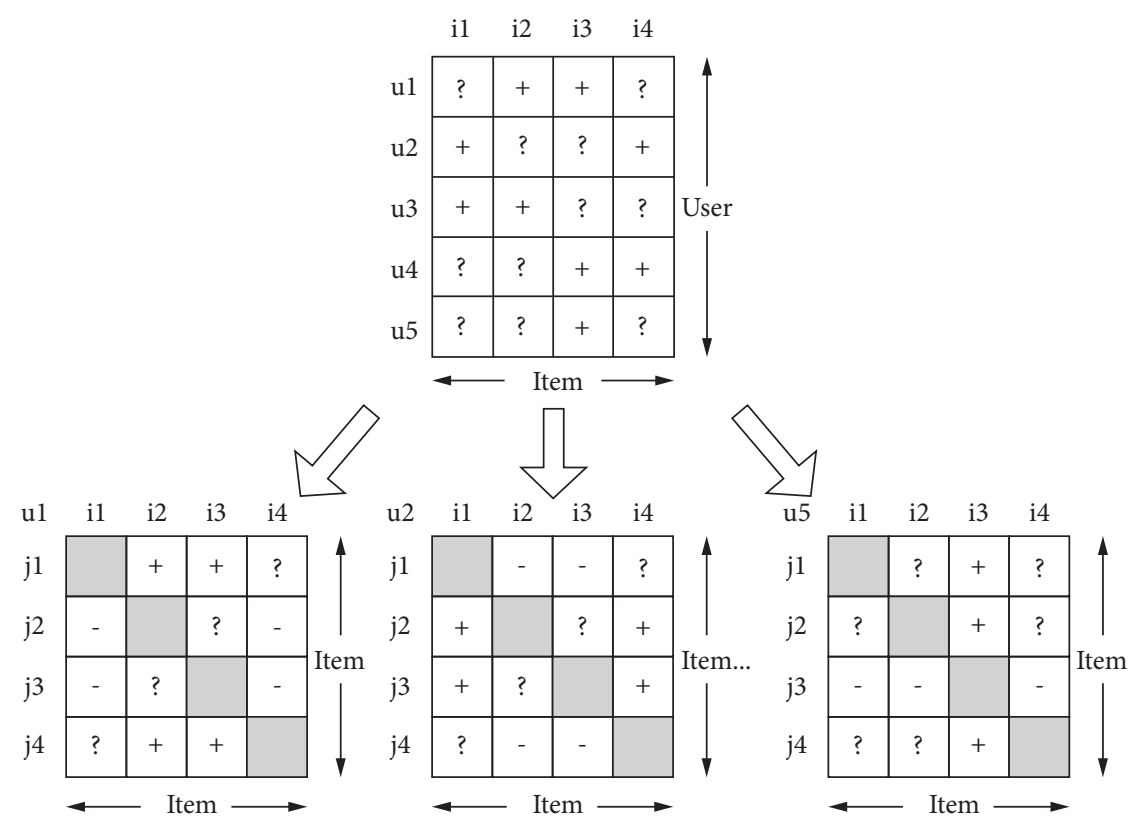

FIGURE 5: Triplet generation process.

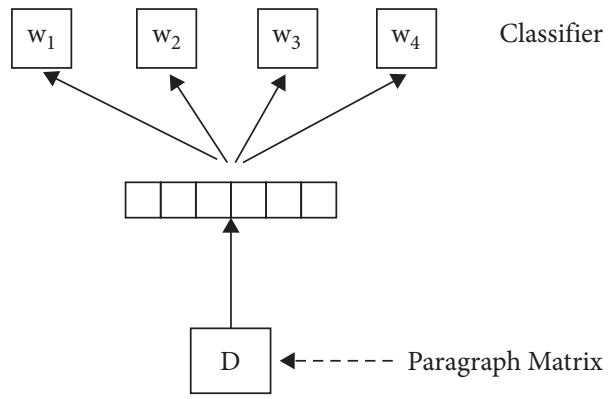

Paragraph id

FIGURE 6: Distributed bag of words of paragraph vectors. 

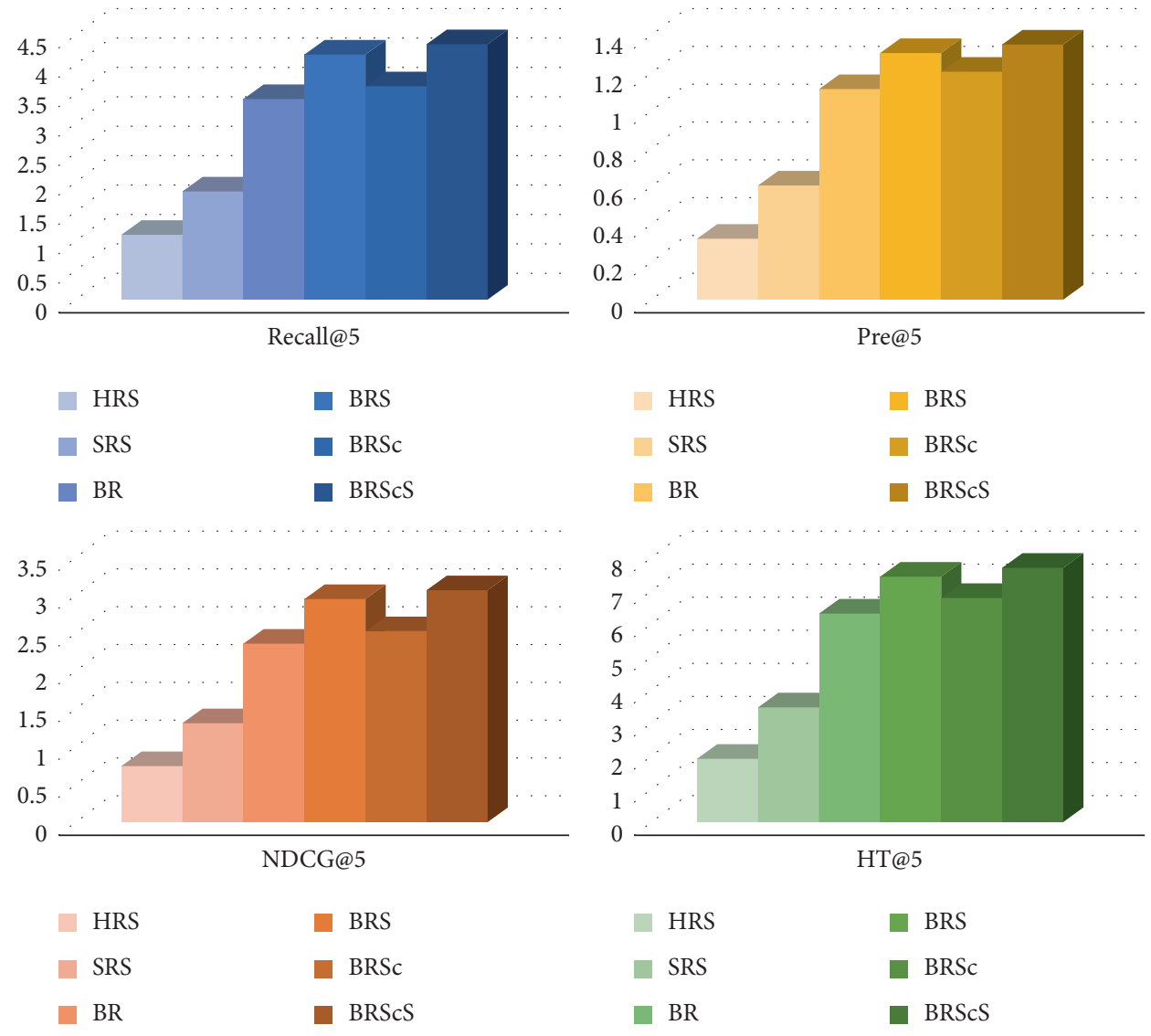

FIGURE 7: Indicators of top-5 recommendation.
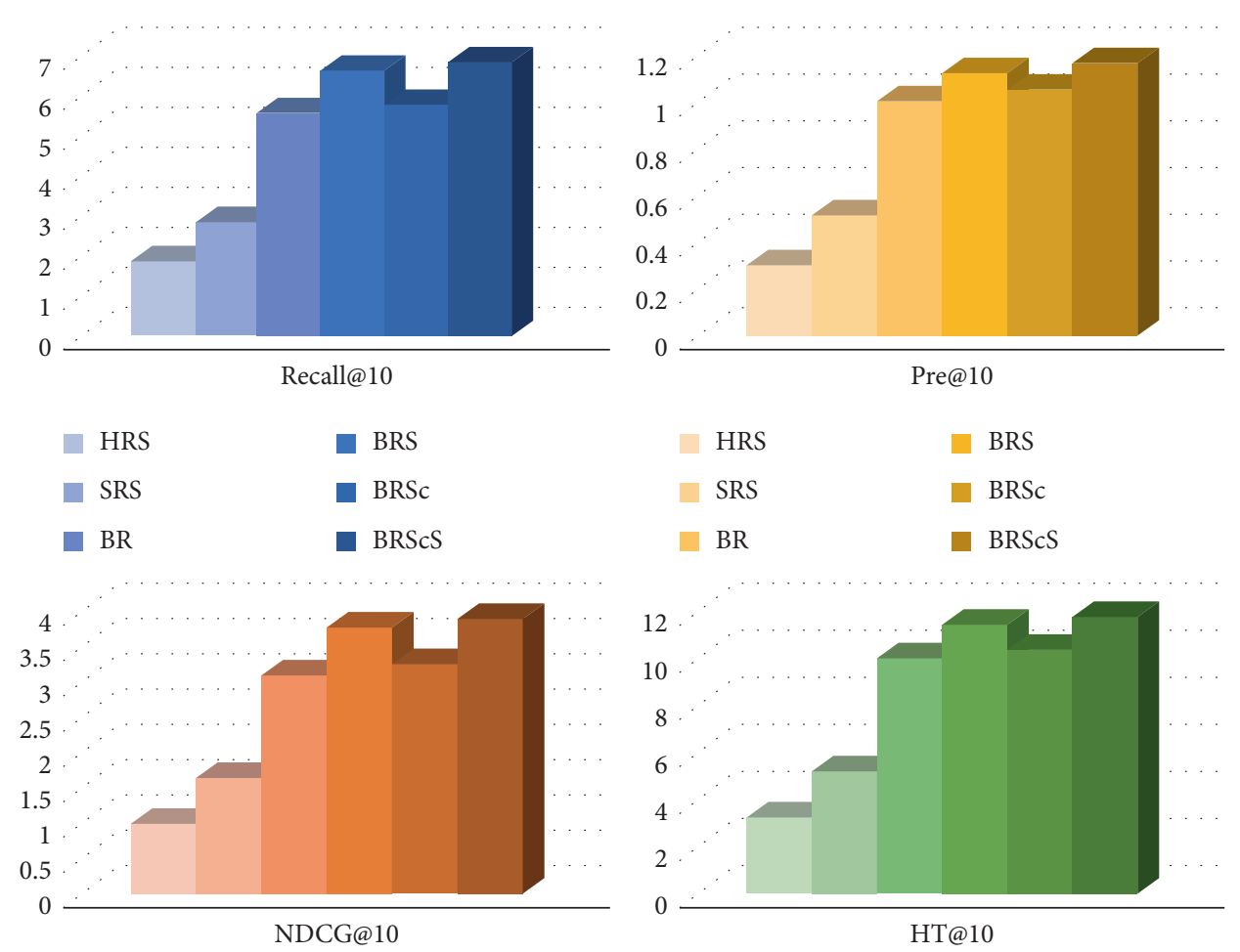
- HRS
- BRS
- HRS
- BRS
SRS
- BRSC
- SRS
- BRSc
BR
- BRScS
- BR
BRScS

Figure 8: Indicators of top-10 recommendation 


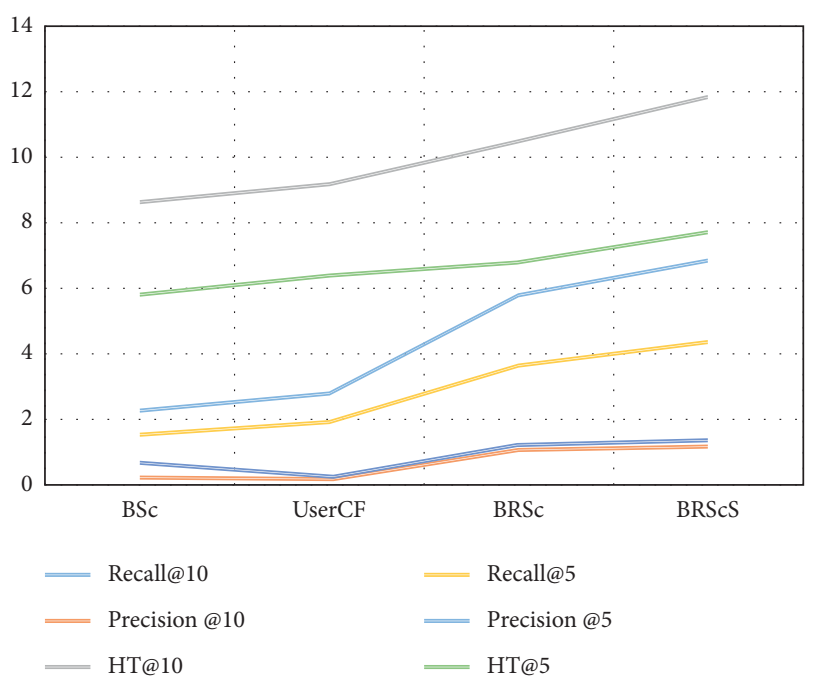

Figure 9: Indicators of Experiment 2.

\section{Conclusions}

BRSc can be distinguished from BSc and users by no more than three indicators (recall, precision, and HT). It examines three different algorithms for social and review-based recommendation: BRS, HRS, and SRS. Based on these findings, we may conclude that incorporating the BPR framework into our model is a sensible decision. These findings show that including social networks in the recommendation process may improve its accuracy. It is clear from all of these models that the BRScS model has the best accuracy, recall, and NDCG scores. In this case, integrating reviews and recommendations indicates the value of doing so. We recommend using BRcS for all indicators. It demonstrates that deep learning-based fusion outperforms the traditional strategy based on collective filtering. Our algorithm is capable of incorporating data from a variety of sources, including scores, reviews, and social networks, to deliver relevant recommendations. Cold start challenges and data scant worries can be alleviated with the use of social network information gleaned from friends' direct and indirect data. $[26,27,28,29]$.

\section{Data Availability}

The data used to support the findings of this study are available from the corresponding author upon request.

\section{Conflicts of Interest}

The authors declare that they have no conflicts of interest.

\section{References}

[1] C. Maddumage, D. Senevirathne, I. Gayashan, T. Shehan, and S. Sumathipala, "Intelligent Recruitment system," in Proceedings of the 2019 IEEE 5th International Conference for Convergence in Technology (I2CT), March 2019.

[2] P. K. Roy, S. S. Chowdhary, and R. Bhatia, "A machine learning approach for automation of Resume recommendation system," Procedia Computer Science, vol. 167, no. 2019, pp. 2318-2327, 2020.

[3] L. Matos Pombo, "Landing on the right job: a machine learning approach to match candidates with jobs applying semantic embeddings," 2019, http://hdl.handle.net/10362/ 60405 .

[4] H. Jelodar, Y. Wang, R. Orji, and S. Huang, "Deep sentiment classification and topic Discovery on Novel Coronavirus or COVID-19 Online discussions: NLP using LSTM Recurrent neural network approach," IEEE Journal of Biomedical and Health Informatics, vol. 24, no. 10, pp. 2733-2742, 2020.

[5] M. Al-Ramahi and I. Alsmadi, "Using data analytics to filter Insincere Posts from Online social networks A case study: Quora Insincere questions," in Proceedings of the 53rd Hawaii International Conference on Systems Science, Maui, HI, USA, 2020.

[6] H. E. Merabet and A. Hajraoui, "A survey of malware detection techniques based on machine learning," International Journal of Advanced Computer Science and Applications, vol. 10, no. 1, pp. 366-373, 2019.

[7] O. Gireesha and L. V Reddy, "An approach to malware analysis and reporting by machine learning," 2016.

[8] J. Kalita and V. Emilia, Advances in Intelligent Systems and Computing 740 Recent Developments in Machine Learning and Data Analytics, Springer, Berlin, Germany, 2018.

[9] L. M. Rojas-Barahona, "Deep learning for sentiment analysis," Language and Linguistics Compass, vol. 10, no. 12, pp. 701-719, 2016.

[10] K. Homsapaya and K. Budsara, "Machine learning for Older Jobseeker and Employment matching," in Proceedings of the 2020 17th International Conference on Electrical Engineering/ Electronics, Computer, Telecommunications and Information Technology (ECTI-CON), pp. 427-430, IEEE, Phuket, Thailand, June 2020.

[11] D. Li, Using Twitter Stream Data for Real-Time Influenza-like Illness Detection and Prediction, pp. 2008-2011, 2015.

[12] P. Sharma and J. Arora, "A review on malware detection schemes using machine learning techniques," Int. J. Eng. Dev. Res.vol. 4, no. 2, pp. 2321-9939, 2016, [Online]. Available:.

[13] A. Basit, ICC T20 Cricket World Cup 2020 Winner Prediction Using Machine Learning Techniques, 2020.

[14] M. Al-Ramahi and I. Alsmadi, "Using data analytics to filter Insincere Posts from Online social networks A case study: Quora Insincere questions," Proceedings of the Annual Hawaii International Conference on System Sciences, vol. 3, pp. 2489-2497, 2020.

[15] D. Yogeshwaran and N. Yuvaraj, Text Classification Using Recurrent Neural Network in Quora, pp. 638-642, 2019.

[16] B. Doshi, "Implementation of Online Portal for CV analysis using KNN algorithm," International Journal for Research in Applied Science and Engineering Technology, vol. 7, no. 4, pp. 1435-1439, 2019.

[17] J. Zhou, X. Hong, and P. Jin, "Information fusion for multisource material data: Progress and challenges," Applied Sciences, vol. 9, no. 17, pp. 3473-17, 2019.

[18] Y. Liu, "Multi-source heterogeneous data fusion based on perceptual semantics in narrow-band Internet of Things," Personal and Ubiquitous Computing, vol. 23, no. 3-4, pp. 413-420, 2019.

[19] L. Zhang, Y. Xie, L. Xidao, and X. Zhang, "Multi-source heterogeneous data fusion," in Proceedings of the 2018 International Conference on Artificial Intelligence and Big Data (ICAIBD), pp. 47-51, 2018. 
[20] Z. Shi, Y. Sun, L. Xiong, Y. Hu, and B. Yin, "A multisource heterogeneous data fusion method for pedestrian tracking," Mathematical Problems in Engineering, vol. 2015, pp. 1-10, 2015.

[21] A. Dhakal, A. Poudel, S. Pandey, S. Gaire, and H. P. Baral, "Exploring deep learning in semantic question matching," in Proceedings of the 2018 IEEE 3rd International Conference on Computing, Communication and Security (ICCCS), pp. 86-91, 2018.

[22] S. T. Gopalakrishna and V. Varadharajan, "Automated tool for Resume classification using Sementic analysis," International Journal of Artificial Intelligence \& Applications, vol. 10, no. 01, pp. 11-23, 2019.

[23] S. Reddy, "Detecting experts on quora by their activity, quality of answers pdf," 2015.

[24] K. V. Deshpande, S. Pan, and J. R. Foulds, "Mitigating Demographic bias in AI-based Resume filtering," in Proceedings of the Adjunct Publication of the 28th ACM Conference on User Modeling, Adaptation and Personalization, pp. 268-275, 2020.

[25] N. Bukarina, "Applying machine learning to a job-candidate matching problem," 2014.

[26] J. Zhang, "Multi-source remote sensing data fusion: Status and trends," International Journal of Image and Data Fusion, vol. 1, no. 1, pp. 5-24, 2010.

[27] M. Adeel Azam, K. Bahadar Khan, M. Ahmad, and M. Mazzara, "Multimodal medical image registration and fusion for quality Enhancement," Computers, Materials \& Continua, vol. 68, no. 1, pp. 821-840, 2021.

[28] S. He, J. Zhang, Y. Cheng, X. Wan, and B. Ran, "Freeway Multisensor data fusion approach integrating data from cellphone Probes and fixed sensors," Journal of Sensors, vol. 2016, pp. 1-13, 2016.

[29] J. Jiang, B. Sheng, and M. Yang, "Research on owa based multi-source heterogeneous data fusion," Journal of Sensors, vol. 11, no. 3, pp. 232-239, 2011.

[30] E. A. Ariz-i, "BRS c S: a hybrid recommendation model fusing multi-source heterogeneous data," EURASIP Journal on Wireless Communications and Networking volume, vol. 2020, p. 124, 2020. 